\title{
MEK inhibitor PD0325901 and vitamin C synergistically induce hypomethylation of mouse embryonic stem cells
}

\author{
Cuiping Li ${ }^{1}$, Baodong Liu ${ }^{1}$, Shangwei Zhong ${ }^{2}$, Hailin Wang ${ }^{1}$ \\ ${ }^{1}$ The State Key Laboratory of Environmental Chemistry and Ecotoxicology, Research Center for Eco-Environmental Sciences, \\ Chinese Academy of Sciences, Beijing 100085, China \\ ${ }^{2}$ University of Chinese Academy of Sciences, Beijing 100039, China
}

Correspondence to: Hailin Wang, email: hlwang@rcees.ac.cn

Keywords: vitamin C, PD0325901, hypomethylation, embryonic stem cells, Prdm14

Received: February 28, 2016

Accepted: April 16, 2016

Published: May 18, 2016

\section{ABSTRACT}

A rationally selected combination of small-molecule chemicals can affect cell plasticity and fate, suggesting an open chemistry way to manipulate cells to achieve a specific goal. Here we for the first time demonstrate that a combination of vitamin $C(\mathrm{Vc})$ and PD0325901 can achieve about $90 \%$ erasure of 5 -methylcytosine $(5 \mathrm{mC})$ within 5 days (decreasing from 3.2 to $0.35 \mathrm{mC}$ per $100 \mathrm{C}$ ) in mouse embryonic stem cells (ESCs). The hypomethylated level is comparable to that of gonadal primordial germ cells (PGCs), whose pluripotency is closely associated with the global DNA hypomethylation. In contrast, Vc or PD0325901 alone only induces a moderately reduced level of global DNA methylation. Our mechanistic study suggested that PD0325901 elevated expression of Prdm14, which repressed de novo methyltransferase Dnmt3b and its cofactor Dnmt3l at levels of protein, via the mode to eliminate $5 \mathrm{mC}$ from de novo synthesis. By further addition of $\mathrm{Vc}$, the oxidation of $5 \mathrm{mC}$ as catalyzed by Tet1/Tet2 dioxygenases was significantly increased as manifested by the elevated level of 5-hydroxymethylcytosine. However, by the depletion of Tet1/Tet2, Vc failed to enhance PD0325901-stimulated hypomethylation of ESCs' genomic DNA. Furthermore, mouse ESCs in Vc/PD0325901-supplemented medium show great morphology and pluripotency. Therefore, we demonstrate a novel and synergistic chemical approach for promoting hypomethylation and sustaining pluripotency of ESCs.

\section{INTRODUCTION}

Mammalian cells demonstrate an amazing plasticity that allows them to develop from one type to the other functionally distinct cells. Embryonic stem cells (ESCs) are just such cells. ESCs are derived from the inner cell mass (ICM) of a developing blastocyst [1]. These cells are in a pluripotent state and are capable of forming all somatic cell lineages and germ cells [2]. Establishment of ESCs can provide the chance to investigate the developmental processes in vitro [3]. Furthermore, due to their pluripotency, various cell types can be generated for regenerative medicine [3].

$5 \mathrm{mC}$ is the product of the methylation of cytosine at carbon-5 position, which is catalyzed by DNA methyltransferases, DNMT1, DNMT3a and DNMT3b, in mammalian cells. In somatic cells, $5 \mathrm{mC}$ (combined with other proteins) functions in regulation of gene expression, $\mathrm{X}$-chromosome inactivation and gene imprinting [4-7]. Interestingly, global erasure of genomic $5 \mathrm{mC}$ occurs in ESCs in blastocysts [8], and the hypomethylation in ESCs is closely linked with pluripotent state [9]. However, unlike ESCs in blastocysts, serum-cultured ES cells often exhibit global hypermethylation [9].

Many studies have shown that a combination of small-molecule chemicals can guide cell fates. As manifested by recent discovery of full chemically induced pluripotent stem cells, neural progenitor cells, and cardiomyocytes [10-12], small-molecule compounds show promising applications in reprogramming and trans-differentiation and the potentials in clinical developments. 
Here, we present a chemical approach to rapidly and effectively promote hypomethylation in mouse embryonic stem cells (ESCs) using two small-molecule compounds. It is known that both vitamin $\mathrm{C}[13,14]$ and $2 \mathrm{i}$ (two smallmolecule kinase inhibitors, PD0325901 and CHIR99021) $[9,15]$ can induce a decrease in genomic $5 \mathrm{mC}$, respectively. Interestingly, they exert $5 \mathrm{mC}$ erasure through two distinct mechanisms. Vc enhances demethylation activity, while $2 \mathrm{i}$ repress de novo DNA methylation synthesis. It is reported that the effects of Vc on DNA methylation are greater in $2 \mathrm{i}$ medium relative to that in serum at several gene promoters, however, the mechanism is not unclarified [14]. Here, we attempted to explore a synergistic effect of $\mathrm{Vc}$ and $2 \mathrm{i}$ on the erasure of genomic $5 \mathrm{mC}$ in mouse ESCs grown in FBS medium. Furthermore, we will investigate which factor in $2 \mathrm{i}$ contributes to the combined DNA demethylation and unveil the mechanisms of action.

\section{RESULTS AND DISCUSSION}

\section{$2 i$ and Ve synergistically induced more pronounced DNA hypomethylation of mouse ESCs}

By the use of highly sensitive approach of ultrahigh performance liquid chromatography-triple quadrupole mass spectrometry coupled with multiple-reaction monitoring (UHPLC-MRM-MS/MS) [13], we observed that the co-treatment of $\mathrm{Vc}$ and $2 \mathrm{i}$ can dramatically reduce $5 \mathrm{mC}$ content of mouse ESCs. After 11 days-treatment, the level of $5 \mathrm{mC}$ was sustained at $0.33 \pm 0.01$ per $100 \mathrm{C}$, indicating a loss of about $90 \% 5 \mathrm{mC}$ or a retention of $10 \%$ $5 \mathrm{mC}$ (Figure 1A and 1B). In contrast, without treatment, $5 \mathrm{mC}$ content slightly waved around $3.3 \pm 0.14$ per $100 \mathrm{C}$ during the cultivation period of 26 days (Figure 1B). With an addition of $2 \mathrm{i}$ or $\mathrm{Vc}$ to the basic medium, $5 \mathrm{mC}$ level declined to about $1.3 \pm 0.02$ per $100 \mathrm{C}(\sim 61 \%$ reduction $)$ or $1.4 \pm 0.01$ per $100 \mathrm{C}(\sim 58 \%$ decrease $)$. The results that either $2 \mathrm{i}$ or $\mathrm{Vc}$ induced hypomethylation of mouse ESCs were consistent with previous work [9, 13-15].

We speculated that the synergistic hypomethylation of mouse ESCs induced by co-treatment of $2 \mathrm{i}$ and Vc may be partly related to enhanced DNA demethylation activity. Therefore, we examined genomic $5 \mathrm{mC}$ oxidation products, which function as the intermediates of DNA demethylation. It is known that $5 \mathrm{mC}$ can be oxidized to produce 5-hydroxymethylcytosine $(5 \mathrm{hmC})$, which is catalyzed by Tet family dioxygenases in a Fe (II) and 2-oxoglutaratedependent manner $[16,17]$. $5 \mathrm{hmC}$ in genomic DNA may undergo replication-dependent loss [18]. Alternatively, $5 \mathrm{hmC}$ can be further oxidized to 5 -formylcytosine $(5 \mathrm{fC})$ and 5-carboxylcytosine $(5 \mathrm{caC})$. The latter two bases in genomic DNA can be efficiently excised by thymine DNA glycosylase (TDG) to regenerate unmethylated cytosine, suggesting an active DNA demethylation [19, 20].

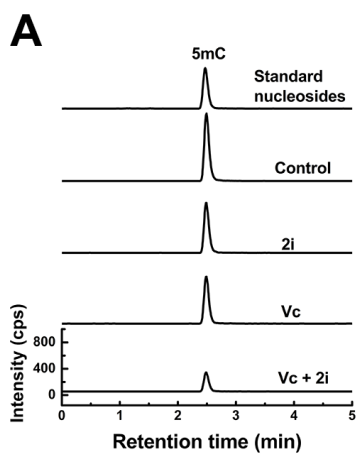

D

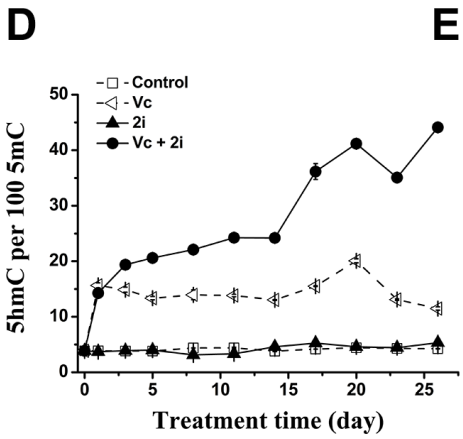

$\mathbf{E}$
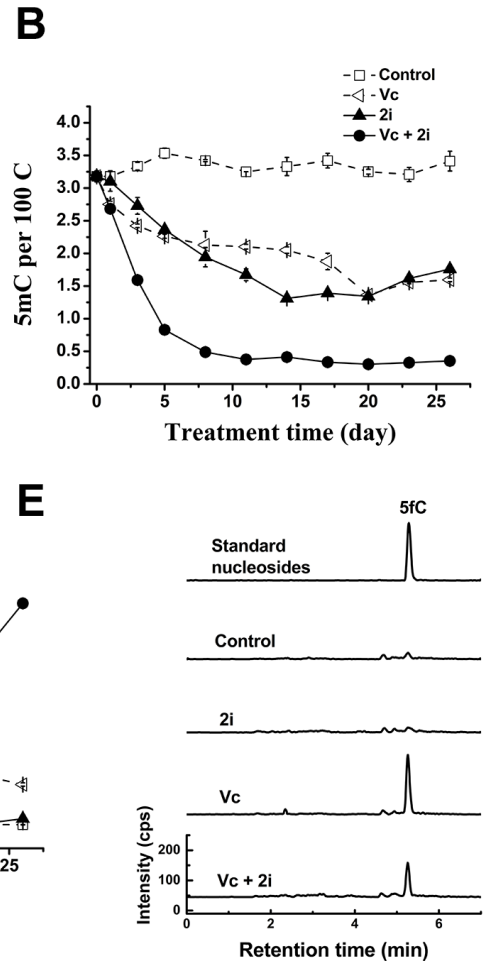

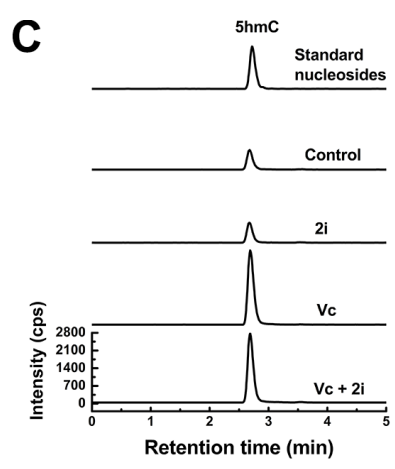

$\mathbf{F}$

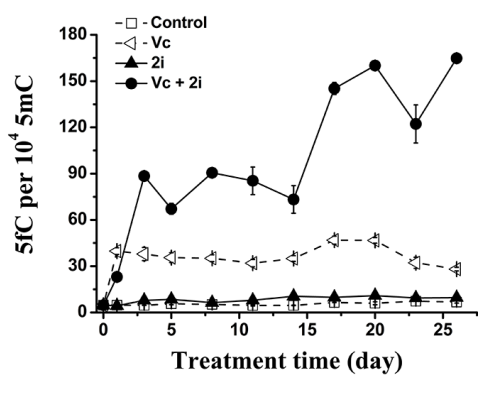

Figure 1: Vc/2i synergistically induced DNA hypomethylation of mouse ESCs. UHPLC-MS/MS detection of $5 \mathrm{mC}$ at 5 day (A), $5 \mathrm{hmC}(\mathbf{C})$ and $5 \mathrm{fC}(\mathbf{E})$ at 1 day after addition of $\mathrm{Vc}$ and $2 \mathrm{i}$ singly or in combination. $5 \mathrm{mC}(5 \mathrm{mC} / \mathrm{C}, \mathbf{B}), 5 \mathrm{hmC}(5 \mathrm{hmC} / 5 \mathrm{mC}, \mathbf{D})$ and $5 \mathrm{fC}$ $(5 \mathrm{fC} / 5 \mathrm{mC}, \mathbf{F})$ frequency time-dependent alteration during 26-day culture of $\mathrm{Vc}$ and $2 \mathrm{i}$ alone or simultaneously. 
As shown in Figure 1C-1F, accompanying with the reduction in $5 \mathrm{mC}$, we also observed that the levels of $5 \mathrm{hmC}$ (Figure 1C and 1D) and its iterative oxidation product $5 \mathrm{fC}$ (Figure $1 \mathrm{E}$ and $1 \mathrm{~F}$ ) increased upon $\mathrm{Vc}$ treatment or $\mathrm{Vc} / 2 \mathrm{i}$ co-treatment. This is consistent with our previous work showing that Vc can enhance Tet activity and promote the generation of $5 \mathrm{hmC}$ [13]. In comparison with $\mathrm{Vc}$ treatment, co-treatment of $\mathrm{Vc}$ and $2 \mathrm{i}$ can further increase the frequency of $5 \mathrm{hmC}$ (44 in per $1005 \mathrm{mC}$ at 26 day) (Figure 1D). We also observed that relative to $\mathrm{Vc}$ treatment alone, the $5 \mathrm{fC}$ frequency further increased when the mouse ESCs were co-treated with Vc and 2i (165 in per $10^{4} 5 \mathrm{mC}$ at 26 day) (Figure 1F). Taken together, these data support that $\mathrm{Vc}$ and $2 \mathrm{i}$ synergistically drove the faster erasure of DNA methylation, partly relating to the enhanced DNA demethylation activity.

\section{Vc/PD0325901 caused demethylation at faster kinetics than $\mathrm{Vc} / 2 \mathrm{i}$}

Next question is which component of $2 \mathrm{i}$ (a combination of PD0325901 and CHIR99021) [21] contributes to the global loss of $5 \mathrm{mC}$ in genomic DNA of mouse ESCs. To answer this question, we tested the combination of $\mathrm{Vc}$ with either PD0325901 (PD) or CHIR99021 (CH).

As shown by Figure $2 \mathrm{~A}$ and $2 \mathrm{~B}$, similar to the co-treatment of Vc and 2i, the co-treatment of PD0325901 and $\mathrm{Vc}$ can induce global and dramatic reduction of genomic $5 \mathrm{mC}$, but not the co-treatment of $\mathrm{Vc}$ and CHIR99021. Interestingly, addition of CHIR99021 slightly suppressed Vc-stimulated demethylation (Figure 2B), whereas Vc/PD0325901 treatment exhibited faster kinetics of $5 \mathrm{mC}$ loss than $\mathrm{Vc} / 2 \mathrm{i}$ co-treatment during 26-day culture of ESCs (Figure 2C). At 11 day, $5 \mathrm{mC}$ level was comparable between $\mathrm{Vc} / \mathrm{PD} 0325901$ and $\mathrm{Vc} / 2 \mathrm{i}$ treatment. The insert in Figure $2 \mathrm{C}$ showed the variation tendency of $5 \mathrm{mC}$ after 11 days. The obtained minimum levels of genomic $5 \mathrm{mC}$ are $0.33 \pm 0.01$ per $100 \mathrm{C}$ and $0.26 \pm 0.01$ per $100 \mathrm{C}$ for the co-treatment of $\mathrm{Vc} / 2 \mathrm{i}$ and $\mathrm{Vc} / \mathrm{PD} 0325901$, respectively. Consistently, Vc/PD0325901 also showed a higher 5hmC frequency (42 in per $1005 \mathrm{mC}$ at 5 day, Figure 2D), due to the combination of Vc-promoted $5 \mathrm{hmC}$ increase and Vc/PD0325901-induced loss of 5mC. These results clearly prove that PD0325901 but not CHIR99021 in two small-molecule kinase inhibitors (2i) contributes to reduced level of genomic $5 \mathrm{mC}$ in ESCs. Regarding the reason that $\mathrm{Vc} / \mathrm{PD} 0325901$ drove a faster $5 \mathrm{mC}$ loss than $\mathrm{Vc} / 2 \mathrm{i}$, we suspect that it is due to slight inhibition of CHIR99021 on demethylation. Since CHIR99021 can inhibit pluripotency-related GSK3 $\beta$ pathway [21], this also suggests that currently and previously observed $2 \mathrm{i}$-induced hypomethylation of ESCs is not pertinent to the inhibition of GSK3 $\beta$ pathway.

Essentially, the Vc/PD0325901 co-treatment induces a loss of $92.1 \%$ genomic $5 \mathrm{mC}$. In other words, only $7.9 \%$ genomic $5 \mathrm{mC}(0.26 \pm 0.01$ per $100 \mathrm{C})$ is resistant to the enhanced $5 \mathrm{mC}$ erasure (Figure $2 \mathrm{C}$ ). The question is that which genes are retained in $\mathrm{Vc} /$ PD0325901-induced erasure of methylation. Studies have shown that imprinted genes, intracisternal A particles and major satellites remained relatively resistant to $2 \mathrm{i}$-induced loss of $5 \mathrm{mC}$ [9]. Similarly, imprinted regions and intracisternal A particle retroelements were also resistant to vitamin $\mathrm{C}$-induced demethylation in N2B27based $2 \mathrm{i}$ medium [14]. Probably these imprinted regions and intracisternal A particles are also resistant to $\mathrm{Vc} /$ PD0325901-induced erasure of methylation. However, to exactly identify the resistant genes will require further genome-wide sequencing of $5 \mathrm{mC}$ and $5 \mathrm{hmC}$.

\section{Tet-dependent DNA demethylation promoted by vitamin $C$ contributed to global hypomethylation induced by PD0325901/Vc}

We next asked whether Tet family dioxygenases contributed to the synergistic hypomethylated state caused by Vc/PD0325901 co-treatment. Regarding the fact that Tet 1 and Tet 2 are two main Tet enzymes expressed in ESCs, we investigated the $5 \mathrm{mC}$ erasure in Tet1/Tet2 depleted ESCs. As shown in Figure 3A and 3B, the depletion of Tet1/Tet 2 abolished the effect of $\mathrm{Vc}$ on DNA methylation. In contrast, by the double knockout of Tet1/Tet2, the treatment of $2 \mathrm{i}$ or PD0325901 can still reduce $5 \mathrm{mC}$ level progressively within 5 days due to a Tetindependent demethylation mechanism. Furthermore, we found that PD0325901 can trigger more pronounced loss of $5 \mathrm{mC}$ compared to $2 \mathrm{i}$, which explain the discrepancy of $\mathrm{Vc} / \mathrm{PD} 0325901$ and $\mathrm{Vc} / 2 \mathrm{i}$-induced erasure of $5 \mathrm{mC}$ in wild-type ESCs. However, co-treated by $\mathrm{Vc} / 2 \mathrm{i}$ or $\mathrm{Vc} /$ PD0325901 did not lead to a more striking loss in $5 \mathrm{mC}$ relative to $2 \mathrm{i}$ or PD0325901 alone upon deleting Tet1/Tet2. The levels of $5 \mathrm{mC}$ at 5 day are comparable between $2 \mathrm{i}$ $\pm \mathrm{Vc}$ and PD0325901 $\pm \mathrm{Vc}$, which are down to 1.5-2.0 $5 \mathrm{mC}$ per $100 \mathrm{C}$. Moreover, after 5-day co-treatment of Vc/ PD0325901, the level of global $5 \mathrm{mC}$ in Tet1/Tet 2 knockout ESCs (1.3 per $100 \mathrm{C}$, Figure 3) is much higher than that of the wild-type of ESCs (0.33 per $100 \mathrm{C}$, Figure 2B). These results support that the depletion of Tet1/Tet2 not only abolished the demethylation effect of Vc, and also eliminated the synergistic $5 \mathrm{mC}$ erasure caused by the co-treatments of $\mathrm{Vc} / 2 \mathrm{i}$ and $\mathrm{Vc} / \mathrm{PD} 0325901$. Collectively, vitamin $\mathrm{C}$-enhanced Tet1/Tet2 activity contributes to the synergistic demethylation of Vc/2i and Vc/PD0325901.

\section{PD0325901 upregulated Prdm14, accompanying with downregulation of Dnmt3b and Dnmt3l, but not Dnmt3a}

Since PD0325901 cannot induce $5 \mathrm{mC}$ erasure through Tet-mediated demethylation, we further examined the protein expression of DNA methyltransferases 
(Dnmt1, Dnmt3a, and Dnmt3b) and related regulation genes (Dnmt31 and Prdm14) [9, 22, 23]. As shown by western blot analysis, Prdm14 was considerably upregulated only in PD0325901-containing treatment (Figure 4). Meanwhile, Dnmt3b and its cofactor Dnmt31, were strongly down-regulated in PD0325901containing treatment. However, the expression level of Dnmt3a, another de novo DNA methyltransferase, was not reduced. The reason is not clear. The maintenance DNA methyltransferase Dnmt1 also showed no change.
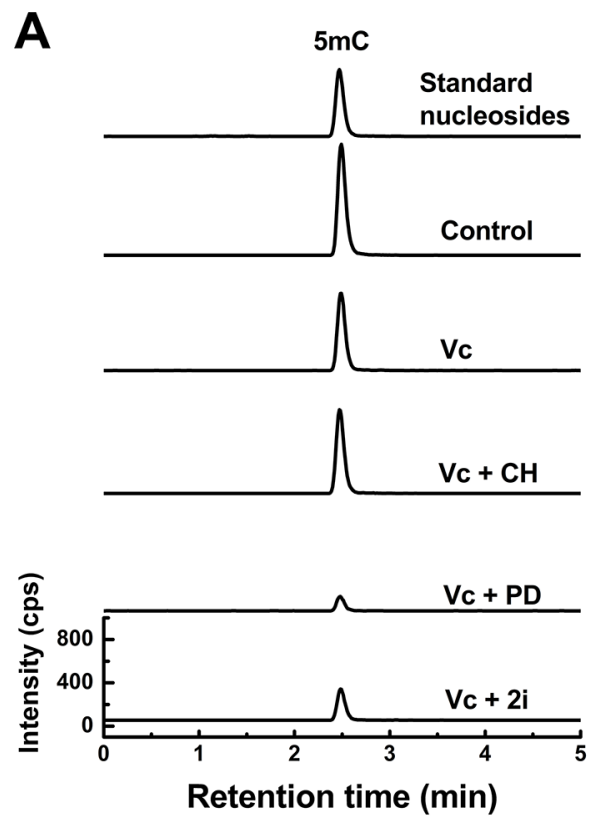

C

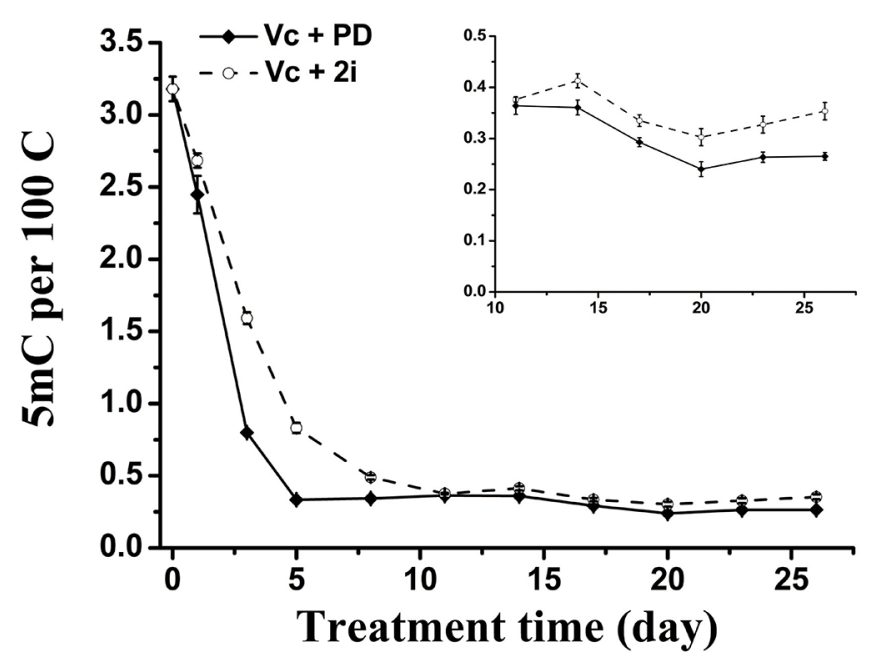

Additionally, Tet1, Tet2 and Tet3, the known three DNA demethylation-related enzymes, were not altered significantly at the protein expression levels (Figure 4). Recent studies have suggested that Prdm14 repressed the expression of de novo DNA methyltransferases Dnmt3a, Dnmt3b and their cofactor Dnmt31 by recruiting polycomb repressive complex 2 (PRC2) to their promoters and played a key role in maintaining hypomethylation in $2 \mathrm{i}$ conditions $[9,22,24]$. We further confirm the relevance between Prdm14 and loss of

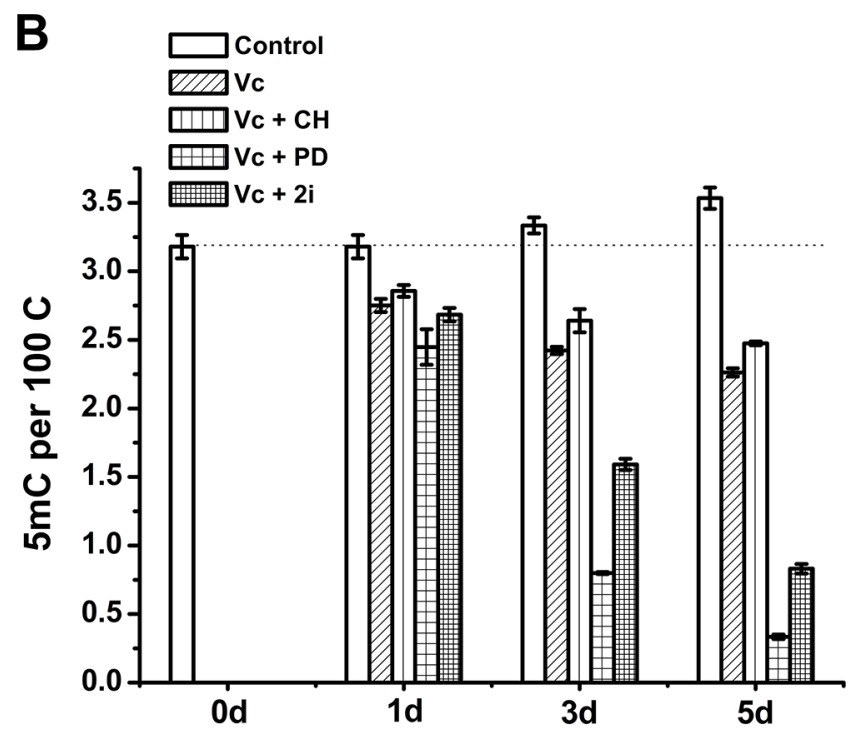

D

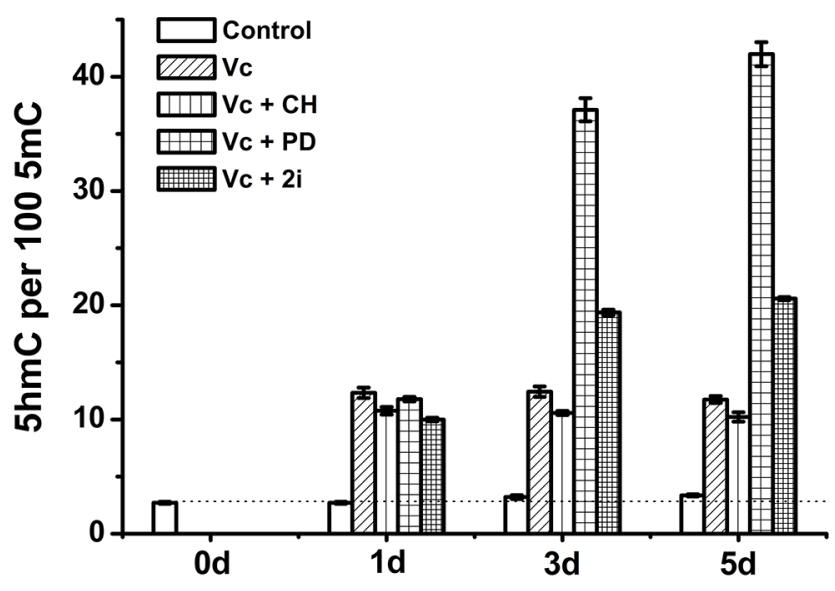

Figure 2: Vc/PD0325901 led to more striking loss of DNA methylation than Vc/2i. UHPLC-MS/MS analysis of $5 \mathrm{mC}$ of mouse ESCs at 5 day (A) and 5mC time-dependent change within 5 days (B) after treatment with Vc, Vc/PD0325901, Vc/CHIR99021 and Vc/2i, respectively. (C) Comparison of decline kinetics in $5 \mathrm{mC}$ between $\mathrm{Vc} / \mathrm{PD} 0325901$ and $\mathrm{Vc} / 2 \mathrm{i}$ during 26 days of treatment. The insert showed the variation trend of $5 \mathrm{mC}$ after 11 days. (D) $5 \mathrm{hmC}$ frequency $(5 \mathrm{hmC} / 5 \mathrm{mC})$ change within 5 days after Vc co-treatment with PD0325901 or CHIR99021 or $2 \mathrm{i}$. 


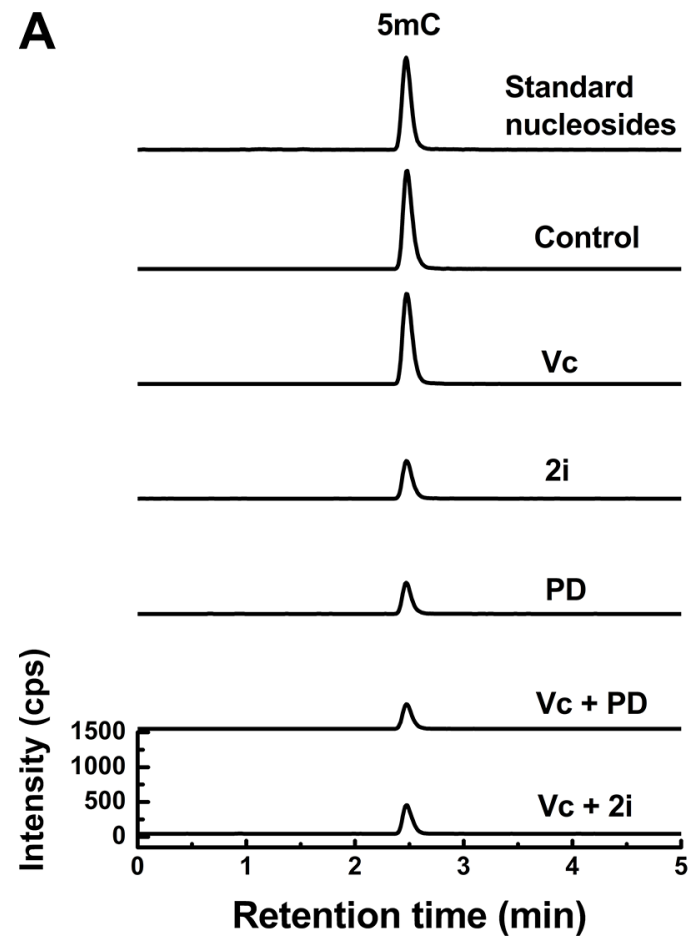

B

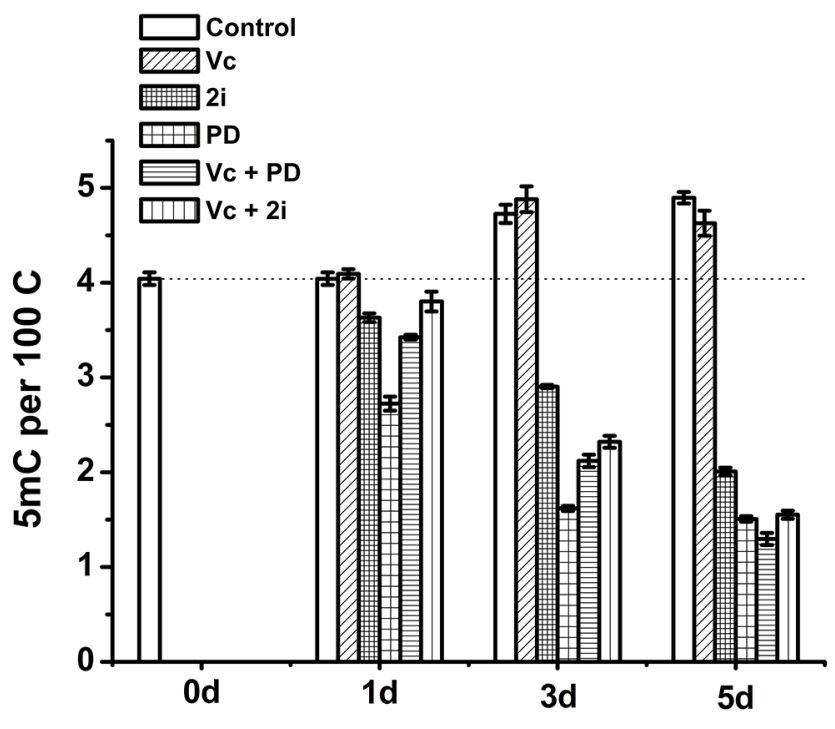

Figure 3: Tet1/Tet2 double knockout attenuated the loss of $5 \mathrm{mC}$ induced by Vc/PD0325901 and Vc/2i relative to that in wild-type ESCs. UHPLC-MS/MS analysis of $5 \mathrm{mC}$ at 5 day (A) and measured the alteration of $5 \mathrm{mC}$ within 5 days $(\mathbf{B})$ after treatment with Vc, 2i, PD0325901, Vc/PD0325901 and Vc/2i.

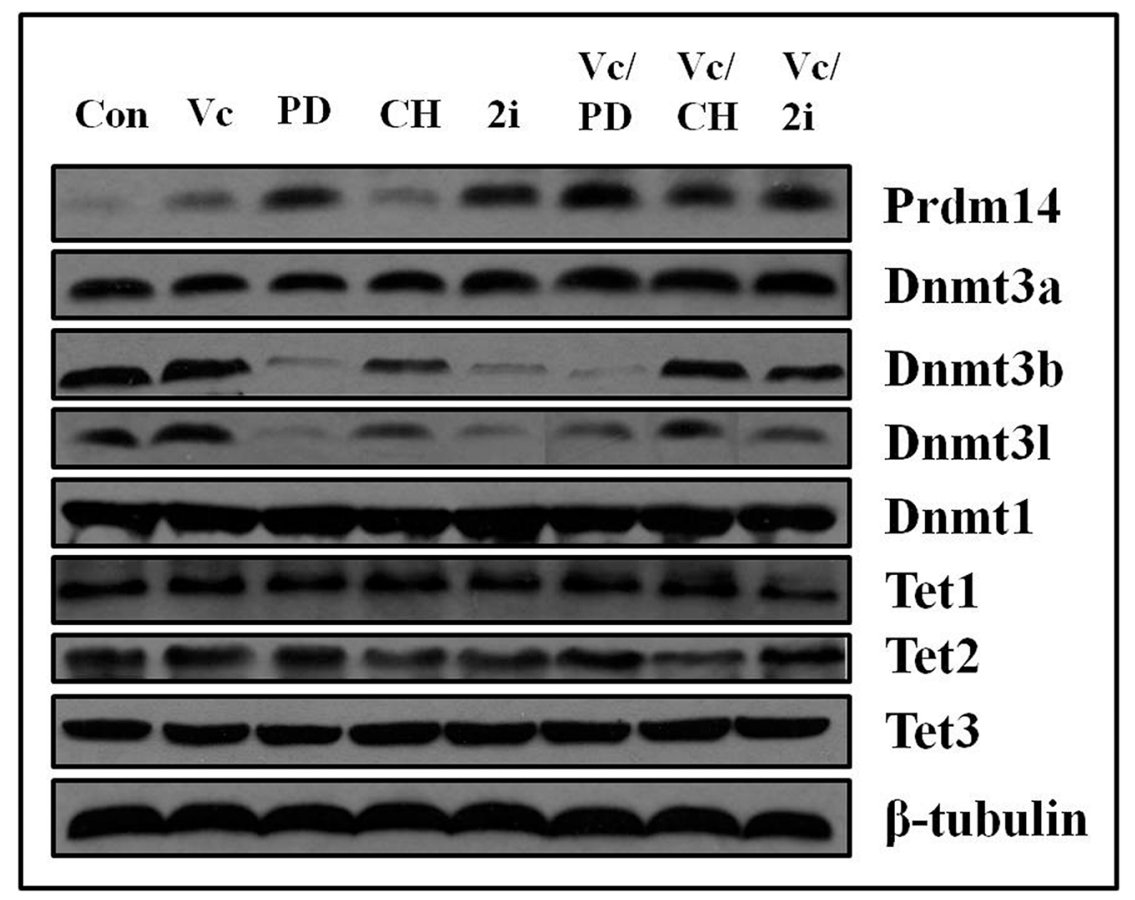

Figure 4: The expression of Prdm14, Dnmt3b and Dnmt3I was altered by PD0325901-containing treatment and Dnmt3a, Dnmt1, Tet1, Tet 2 and Tet 3 exhibited no change in various culture conditions. $\beta$-tubulin was used as the internal reference. The bands from left to right corresponded to the treatment of control, Vc, PD0325901, CHIR99021, 2i, Vc/PD0325901, Vc/ CHIR99021 and Vc/2i, respectively. 
methylation in our Vc/PD0325901 system. Initially, we culture mouse ESCs in basic medium supplemented with Vc/PD0325901 for 5 days to elevate the expression of Prdm14. Next, we downregulate the levels of Prdm14 with siRNA to examine the alteration of Dnmt and $5 \mathrm{mC}$ level. We observed by qPCR that mRNA expression of Prdm14 decreased 31\% after silencing. Following by the downregulation of Prdm14, Dnmt3a and Dnmt3b were elevated by 1.84 -fold $(p<0.001)$ and 2.44 -fold $(p<0.001)$, respectively, and their regulator Dnmt31 was also upregulated by 1.36 -fold (Figure $5 \mathrm{~A}$ ). In contrast, the mRNA level of Dnmt1 kept constant. Accompanying with the downregulation of Prdm14, 5mC content increased by 1.8-fold (Figure 5B). Collectively, these data suggested that PD0325901 promotes the expression of Prdm14, which further down-regulates Dnmt3b and Dnmt31. By the

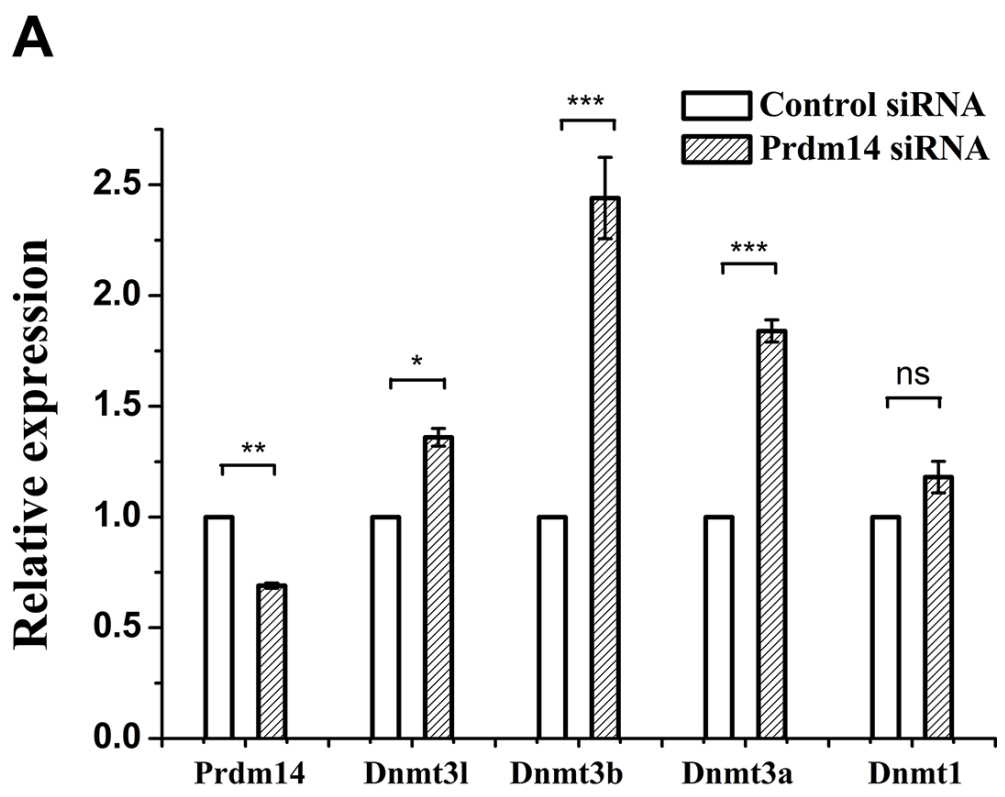

B

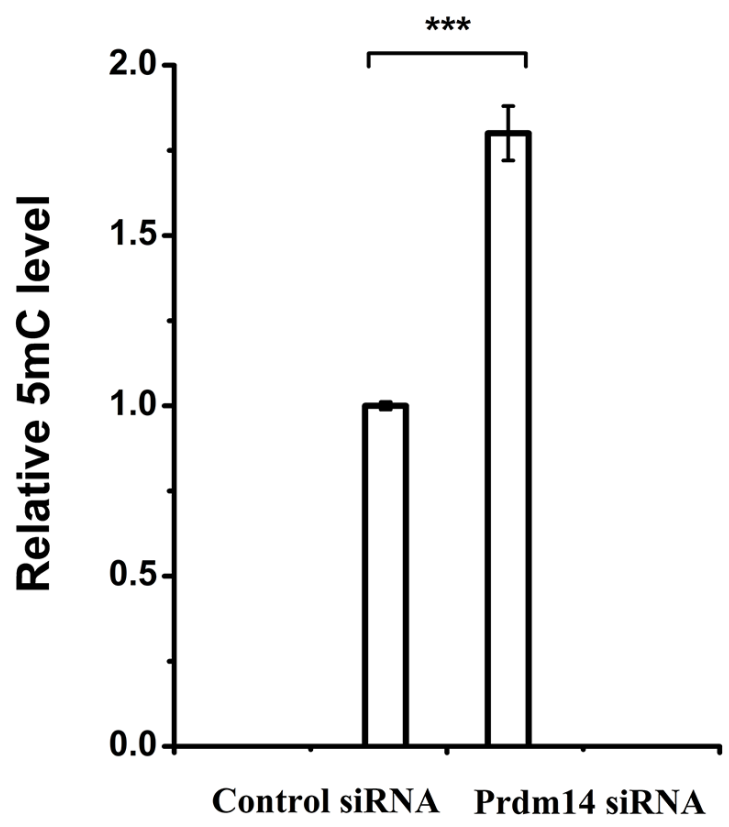

Figure 5: The effect of Prdm14 on Dnmt and DNA methylation in mouse ESCs. (A) qPCR analysis of Prdm14 and Dnmt mRNA expression levels after the downregulation of Prdm14 in comparison with control siRNA. Error bars show s.d. Unpaired student's $t$-test was used to assess the statistical significance. ${ }^{*} P<0.05, * * P<0.01, * * * P<0.001$, ns represents no statistical significance. (B) UHPLC-MS/MS analysis of global 5mC levels after knockdown of Prdm14 compared to control siRNA. 
mechanism, de novo synthesis of genomic $5 \mathrm{mC}$ is greatly reduced.

\section{The effect of Ve/PD0325901 on pluripotency of mouse ESCs}

As we show, co-treatment of Vc and PD0325901 can induce global hypomethylation of mouse ESCs within 5 days, however, it is not known whether pluripotency can be maintained under these conditions. To answer this question, we tested mRNA expression of several core pluripotency factors which have been functionally validated as playing vital roles [25]. The results revealed that expression levels of Oct4, SOX2, Esrrb and Sall4 remained constant after 5 days of $\mathrm{Vc} / \mathrm{PD} 0325901$ treatment relative to culture in serum (Figure 6A). Nanog and Tcf3 showed 1.3-fold and 1.6-fold increase, respectively, while Klf4 was observed by $42 \%$ decrease and Rex 1 only exhibited about $12 \%$ downregulation. The results indicated pluripotency factors showed diverse alteration of expression in Vc/PD0325901 co-treatment, however, the expression of most core factors, Oct4 and SOX2, was relatively uniform. Recent studies have suggested that pluripotency genes expression is not changed by $\mathrm{Vc}$, whereas $2 \mathrm{i}$ increases expression of Nanog [14]. Therefore, alteration of pluripotency genes expression induced by Vc/PD0325901 should mainly be attributed to the action of PD0325901.

We next examine the pluripotency using alkaline phosphatase (AP) assay. Through AP staining assay, we can monitor ESCs undifferentiation or differentiation. After continuous culture of mouse ESCs in $\mathrm{Vc} /$ PD0325901-supplemented medium for 26 days, as shown in Figure 6B (b), we observed that ESCs were well stained and ball-like without outgrowth, that is, cells remained an undifferentiated state. In contrast, ESCs grown in serum for 26 days showed the purple-black and balllike state in part, while others exhibited light color with spread (Figure 6B (a)), indicating that ESCs in serum showed heterogeneity in morphology and pluripotency, in line with other reports [15]. The results revealed that PD0325901 together with Vc can maintain ES cells in great morphology and an undifferentiated state.

In summary, we demonstrated a synergistic mechanism of enhancing DNA demethylation and repressing DNA methylation simultaneously in ESCs and

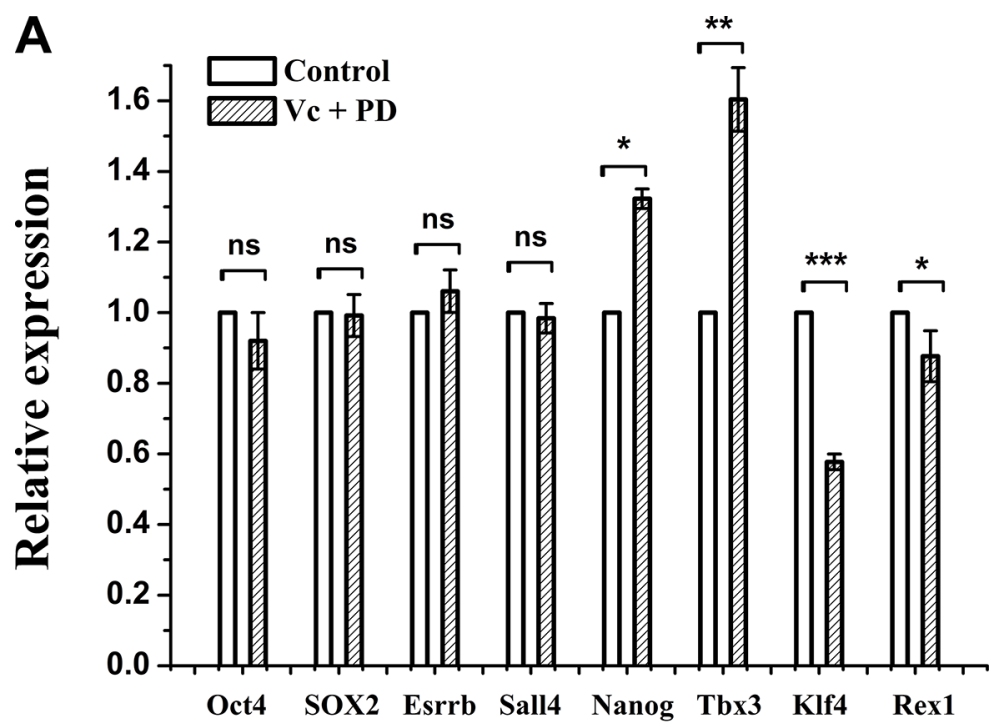

B

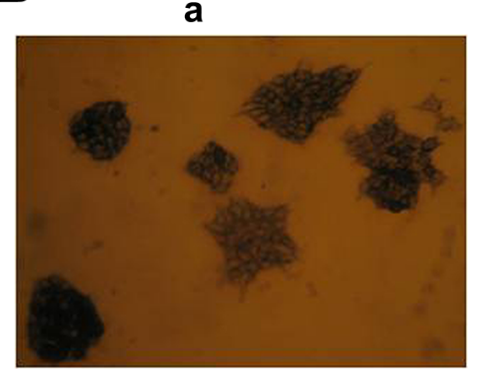

b

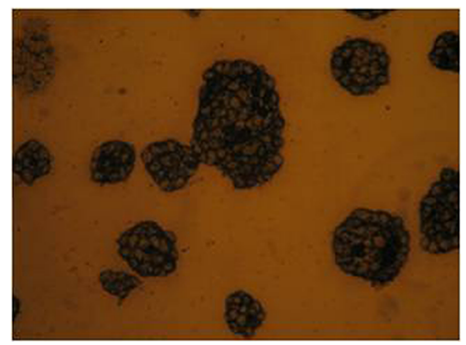

Figure 6: The effect of Vc/PD0325901 on pluripotency-related genes and alkaline phosphatase. (A) Expression of partial pluripotency genes was changed at 5 day of Vc/PD0325901 treatment relative to FBS untreated sample. Data were represented as mean \pm s.d. (B) Mouse ESCs maintained in Vc/PD0325901-supplemented medium showed great morphology and an undifferentiated state (b) relative to ESCs grown in FBS (a) at 26 day. 
developed a novel approach for maintaining ES cells at a hypomethylated and undifferentiated state and in great morphology.

\section{MATERIALS AND METHODS}

\section{Cell culture conditions and treatment}

Mouse embryonic stem cells of wild type (WT mouse ESCs, $129 \mathrm{SvEv}$ ) and of Tet1/Tet2 double knockout $\left(\right.$ Tet $1 /$ Tet $\left.2^{--}\right)$were employed in the assay. Initially, cells were maintained in high-glucose Dulbecco's Modified Eagle Medium (DMEM) (HyClone) supplemented with $20 \%$ ES FBS (Corning), 1000 units $/ \mathrm{ml}$ leukemia inhibitory factor (LIF) (Millipore), $0.1 \mathrm{mM}$ nonessential amino acids (NEAA), $1 \mathrm{mM}$ sodium pyruvate (Gibco), $2 \mathrm{mM}$ L-glutamine, $0.1 \mathrm{mM} \beta$-mercaptoethanol, $33 \mathrm{IU} / \mathrm{ml}$ penicillin and $33 \mu \mathrm{g} / \mathrm{ml}$ streptomycin (Corning). We define the medium described above as the basic medium which may be added extra small molecule. For cultures without feeders, cells were grown in $0.1 \%$ gelatincoated dishes and were incubated at $37^{\circ} \mathrm{C}$ in a humid atmosphere with $5 \% \mathrm{CO}_{2}$.

To investigate the effect of small-molecule compounds on the DNA methylation, the basic medium was supplemented with $1.0 \mu \mathrm{M}$ PD0325901 (Stemolecule), $3.0 \mu \mathrm{M}$ CHIR99021 (Stemolecule) and 100 $\mu \mathrm{M}$ vitamin $\mathrm{C}$ alone or in combination. When cells grew to $50 \%$ confluence, fresh medium with small-molecule compounds was used for additional culture for $24 \mathrm{~h}$ or longer. When the exposure time was reached, cells were dissociated with trypsin and then were split into two parts, nine tenths for DNA extraction and another one tenth for further culture in the corresponding culture medium which was changed daily.

\section{DNA extraction and enzymatic digestion}

Genomic DNA extraction was performed with a Genomic DNA Purification Kit (Promega) according to the manufacturer's instructions. The concentration of extracted DNA was determined with NanoDrop 2000 (Thermo Scientific) and quality was evaluated with the ratio of absorbance at $260 \mathrm{~nm}$ and $280 \mathrm{~nm}$.

To obtain mononucleosides, $5 \mu \mathrm{g}$ DNA was digested with $2 \mathrm{U}$ calf intestinal phosphatase, $1 \mathrm{U}$ DNase I and $0.005 \mathrm{U}$ snake venom phosphodiesterase I (New England Biolabs) at $37^{\circ} \mathrm{C}$ overnight. To remove enzymes used for digestion, DNA solution was filtered with ultra-filtration tubes with molecule weight cutoff at $3 \mathrm{KDa}$ (Pall). The filter solution was analyzed with UHPLC-MS/MS for $5 \mathrm{mC}, 5 \mathrm{hmC}$ and $5 \mathrm{fC}$.

\section{UHPLC-MS/MS analysis of $5 \mathrm{mC}, 5 \mathrm{hmC}$ and $5 \mathrm{fC}$}

The UHPLC-MS/MS analysis was performed according to the published methods [13] with a minor modification. The Agilent 1290 UHPLC system and Zorbax Eclipse Plus C18 column (100 mm $\times 2.1 \mathrm{~mm}, 1.8$ $\mu \mathrm{m}$, Agilent Technologies) were adopted for $5 \mathrm{mC}, 5 \mathrm{hmC}$ and $5 \mathrm{fC}$ analysis. In brief, the mobile phase consisting of $5.0 \%$ methanol and $95 \%$ water with $0.1 \%$ formic acid was applied for $5 \mathrm{mC}$ analysis. An optimized gradient elution was used for $5 \mathrm{hmC}$ and $5 \mathrm{fC}$ analysis: $0-3 \mathrm{~min}$, $5.0 \% \mathrm{~B}$; 3-6min, $15.0 \% \mathrm{~B}$; 6-10 min, $100 \% \mathrm{~B} ; 10-15$ min, $5.0 \% \mathrm{~B}$. Solvent A was $2.0 \mathrm{mM} \mathrm{NH}_{4} \mathrm{HCO}_{3}$ aqueous solution ( $\mathrm{pH}$ 9.0), and solvent B was $100 \%$ methanol. The flow rate was set at $0.25 \mathrm{~mL} / \mathrm{min}$. The elution from the column was directly injected into ESI-G6410B triple quadrupole mass spectrometer. The multiple reaction monitoring (MRM) mode was employed for the experiment, and set as follows: $\mathrm{m} / \mathrm{z} 242 \rightarrow 126$ for $5 \mathrm{mC}$ (collision energy, $5 \mathrm{eV}$ ); $\mathrm{m} / \mathrm{z} 228 \rightarrow 112$ for $\mathrm{dC}(5 \mathrm{eV}) ; \mathrm{m} / \mathrm{z} 258 \rightarrow 142$ for $5 \mathrm{hmC}(5 \mathrm{eV})$ and $\mathrm{m} / \mathrm{z}$ $256 \rightarrow 140$ for $5 \mathrm{fC}(5 \mathrm{eV})$. The capillary and fragment voltages were set at $+3500 \mathrm{~V}$ and $90 \mathrm{~V}$, respectively. Each sample was analyzed three times with an injection volume of $5 \mu$ l. The stable isotopes $5{ }^{\prime}-\left(\right.$ methyl $\left.-\mathrm{d}_{3}\right)-2^{\prime}-$ deoxycytidine ( $\left.\left[\mathrm{D}_{3}\right]\right) 5 \mathrm{mC}$ (Torto Research Chemicals), 5 '-(hydroxymethyl-d $\mathrm{d}_{3}$ )-2'-deoxycytidine $\left(\left[\mathrm{D}_{3}\right]\right) 5 \mathrm{hmC}$ and $\left[{ }^{15} \mathrm{~N}_{3}\right]$-dC (Cambridge Isotope laboratories, Inc.) were used as the internal standard for the calibration of $5 \mathrm{mC}$, $5 \mathrm{hmC}$ and $5 \mathrm{fC}$. The $5 \mathrm{mC}, 5 \mathrm{hmC}$ and $5 \mathrm{fC}$ concentrations were estimated based on the corresponding standard curves.

\section{Western blot analysis}

Whole cell protein extracts were prepared with cold RIPA buffer. Total proteins $(50 \mu \mathrm{g})$ were separated on SDS-PAGE and then transferred to polyvinylidene fluoride (PVDF) membranes. After blocking in 5\% non-fat milk, membranes were incubated with the indicated primary antibody and secondary antibodies before visualization Primary antibodies used were DNMT1 (abcam, ab13537, 1:1000), DNMT3a (bioworld, BS6587, 1:1000), DNMT3b (abcam, ab13604, 1:500), DNMT31 (abcam, ab3493, 1:1000), Prdm14 (bioworld, BS7634, 1:1000), Tet1 (bioworld, BS6966, 1:1000), Tet2 (bioworld, BS7804, 1:1000), Tet3 (abcam, ab135033, 1:700) and $\beta$-tubulin (bioworld, BS1482MH, 1:1500). Secondary antibodies used were goat anti-rabbit IgG (1:5000) and goat antimouse IgG (1:5000).

\section{Gene silencing and real-time quantitative PCR}

Prdm14 mRNA in WT mouse ESCs was silenced by siRNAs purchased from GenePharma. SiRNA transfection was performed by Lipofectamine RNAi MAX according to the manufacturer's instructions and the effect of RNAi was examined after treatment for 3 days. To ensure the efficiency of RNAi, a second transfection was carried out after transfection for 1 day. 
After 3 days of transfection, total RNA was extracted from cells with Trizol reagent (Life technologies Corporation). $1 \mu \mathrm{g}$ RNA was reversely transcribed to cDNA with Promega reverse transcription system. Real-time PCR was performed using Promega GoTaq qPCR Master Mix following the manufacturer's protocol on Stratagene Mx3005P real-time PCR System (Aglient Technologies). The mRNA levels of 5mC-related and pluripotent genes were examined and the relative expression of genes was normalized to the housekeeper gene Gapdh. Two-tailed and unpaired $t$-tests were performed with Graphpad Prism 5 software. Primer sequences were listed as below.

Prdm14 forward: 5'-CAGCGACTTCATTGCCAAA GGAG-3'

Prdm14 reverse: 5'-GCCGTCGATAAAATGGCT CAGG-3'

Dnmt3a forward: 5'-CGCAAAGCCATCTACGAA GTCC-3'

Dnmt3a reverse: 5'- GCTTGTTCTGCACTTCCA

CAGC-3'

Dnmt3b forward: 5'-CGCACAACCAATGACTCT GCTG-3'

Dnmt3b reverse: 5'-GGTGACTTCAGAAGCCAT CCGT-3'

Dnmt1 forward: 5'-GGACAAGGAGAATGCCATG AAGC-3'

Dnmt1 reverse: 5'-TTACTCCGTCCAGTGCCA CCAA-3'

Dnmt31 forward: 5'-CTGTGGAACTCTCCAGGT GTAC-3'

Dnmt31 reverse: 5'- GTGCAGTAACTCTGGTGTC CATC-3' TGT-3'

Gapdh forward: 5'-GTGTTCCTACCCCCAATG

Gapdh reverse: 5'-ATTGTCATACCAGGAAAT GAGCTT-3'

Oct4 forward: 5'-AGTCTGGAGACCATGTTTCT GAAGT-3'

Oct4 reverse: 5'-TACTCTTCTCGTTGGGAATA CTCAATA-3'

SOX2 forward: 5'-CATGAGAGCAAGTACTGG CAAG-3'

SOX2 reverse: 5'-CCAACGATATCAACCTGC ATGG-3'

Nanog forward: 5'-AGGACAGGTTTCAGAAGCA GAAGT-3'

Nanog reverse: 5'-TCAGACCATTGCTAGTCTTC AACC-3'

Klf4 forward: 5'-AGGAGCCCAAGCCAAAG AGG-3'

Klf4 reverse: 5'-CGCAGGTGTGCCTTGAG ATG-3'

Esrrb forward: 5'-CAGGCAAGGATGACAGA CG-3'

Esrrb reverse: 5'-GAGACAGCACGAAGGACT GC-3'
Rex1 forward: 5'-GAGACTGAGGAAGATGGCT TCC-3'

Rex1 reverse: 5'-CTGGCGAGAAAGGTTTTGC TCC-3'

Sall4 forward: 5'-GTTAGATGTCAAGGCCAAGG AC-3' GAT-3'

Sall4 reverse: 5'-GGCGTCTACAGAGAGACTC

Tbx3 forward: 5'-TTATTTCCAGGTCAGGAGATG GC-3' TC-3'

Tbx3 reverse: 5'-GGTCGTTTGAACCAAGTCCC

\section{Alkaline phosphatase staining}

Alkaline phosphatase staining was performed by StemTAG ${ }^{\mathrm{TM}}$ alkaline phosphatase staining and activity assay kit (colorimetric) (Cells Biolabs, Inc.) according to the manufacturer's instructions.

\section{CONFLICTS OF INTEREST}

The authors disclose no potential conflicts of interest.

\section{GRANT SUPPORT}

This study was supported by grants from the National Natural Science Foundation of China (21327006 and 21435008 to H.W.), and the Strategic Priority Research Program of the Chinese Academy of Sciences (XDB14030200 to H.W.).

\section{REFERENCES}

1. Evans MJ, Kaufman MH. Establishment in culture of pluripotential cells from mouse embryos. Nature. 1981; 292:154-156.

2. Smith AG. Embryo-derived stem cells: of mice and men. Annu Rev Cell Dev Biol. 2001; 17:435-462.

3. Murry CE, Keller G. Differentiation of embryonic stem cells to clinically relevant populations: lessons from embryonic development. Cell. 2008; 132:661-680.

4. Bird A. DNA methylation patterns and epigenetic memory. Genes Dev. 2002; 16:6-21.

5. Jones PA, Takai D. The role of DNA methylation in mammalian epigenetics. Science. 2001; 293:1068-1070.

6. Deaton AM, Bird A. CpG islands and the regulation of transcription. Genes Dev. 2011; 25:1010-1022.

7. Wu SC, Zhang Y. Active DNA demethylation: many roads lead to Rome. Nat Rev Mol Cell Biol. 2010; 11:607-620.

8. Smith ZD, Chan MM, Mikkelsen TS, Gu H, Gnirke A, Regev A, Meissner A. A unique regulatory phase of DNA methylation in the early mammalian embryo. Nature. 2012; 484:339-344. 
9. Ficz G, Hore TA, Santos F, Lee HJ, Dean W, Arand J, Krueger F, Oxley D, Paul YL, Walter J, Cook SJ, Andrews S, Branco MR, Reik W. FGF signaling inhibition in ESCs drives rapid genome-wide demethylation to the epigenetic ground state of pluripotency. Cell Stem Cell. 2013; 13:351-359.

10. Hou P, Li Y, Zhang X, Liu C, Guan J, Li H, Zhao T, Ye J, Yang W, Liu K, Ge J, Xu J, Zhang Q, et al. Pluripotent stem cells induced from mouse somatic cells by small-molecule compounds. Science. 2013; 341:651-654.

11. Long Y, Wang M, Gu H, Xie X. Bromodeoxyuridine promotes full-chemical induction of mouse pluripotent stem cells. Cell Res. 2015; 25:1171-1174.

12. Cheng L, Hu W, Qiu B, Zhao J, Yu Y, Guan W, Wang M, Yang W, Pei G. Generation of neural progenitor cells by chemical cocktails and hypoxia. Cell Res. 2015; 25: 645-646.

13. Yin R, Mao SQ, Zhao B, Chong Z, Yang Y, Zhao C, Zhang D, Huang H, Gao J, Li Z, Jiao Y, Li C, Liu S, et al. Ascorbic acid enhances Tet-mediated 5-methylcytosine oxidation and promotes DNA demethylation in mammals. J Am Chem Soc. 2013; 135:10396-10403.

14. Blaschke K, Ebata KT, Karimi MM, Zepeda-Martínez JA, Goyal P, Mahapatra S, Tam A, Laird DJ, Hirst M, Rao A, Lorincz MC, Ramalho-Santos M. Vitamin C induces Tetdependent DNA demethylation and a blastocyst-like state in ES cells. Nature. 2013; 500:222-226.

15. Leitch HG, McEwen KR, Turp A, Encheva V, Carroll T, Grabole N, Mansfield W, Nashun B, Knezovich JG, Smith A, Surani MA, Hajkova P. Naive pluripotency is associated with global DNA hypomethylation. Nat Struct Mol Biol. 2013; 20:311-316.

16. Tahiliani M, Koh KP, Shen Y, Pastor WA, Bandukwala H, Brudno Y, Agarwal S, Iyer LM, Liu DR, Aravind L, Rao A. Conversion of 5-methylcytosine to 5-hydroxymethylcytosine in mammalian DNA by MLL partner TET1. Science. 2009; 324:930-935.
17. Wu H, Zhang Y. Mechanisms and functions of Tet proteinmediated 5-methylcytosine oxidation. Genes Dev. 2011; 25:2436-2452.

18. Inoue A, Zhang Y. Replication-dependent loss of 5-hydroxymethylcytosine in mouse preimplantation embryos. Science. 2011; 334:194.

19. He YF, Li BZ, Li Z, Liu P, Wang Y, Tang Q, Ding J, Jia Y, Chen Z, Li L, Sun Y, Li X, Dai Q, et al. Tet-mediated formation of 5-carboxylcytosine and its excision by TDG in mammalian DNA. Science. 2011; 333:1303-1307.

20. Maiti A, Drohat AC. Thymine DNA glycosylase can rapidly excise 5-formylcytosine and 5-carboxylcytosine: Potential implications for active demethylation of $\mathrm{CpG}$ sites. J Biol Chem. 2011; 286:35334-35338.

21. Ying QL, Wray J, Nichols J, Batlle-Morera L, Doble B, Woodgett J, Cohen P, Smith A. The ground state of embryonic stem cell self-renewal. Nature. 2008; 453: 519-523.

22. Yamaji M, Ueda J, Hayashi K, Ohta H, Yabuta Y, Kurimoto K, Nakato R, Yamada Y, Shirahige K, Saitou M. PRDM14 ensures naive pluripotency through dual regulation of signaling and epigenetic pathways in mouse embryonic stem cells. Cell Stem Cell. 2013; 12:368-382.

23. Hackett JA, Dietmann S, Murakami K, Down TA, Leitch HG, Surani MA. Synergistic mechanisms of DNA demethylation during transition to ground-state pluripotency. Stem Cell Rep. 2013; 1:518-531.

24. Nakaki F, Saitou M. PRDM14: a unique regulator for pluripotency and epigenetic reprogramming. Trends Biochem Sci. 2014; 39:289-298.

25. Nichols J, Smith A. Pluripotency in the embryo and in culture. Cold Spring Harb Perspect Biol. 2012; 4:a008128. 